\title{
ZERNIKE MOMENT-BASED FEATURE EXTRACTION FOR FACIAL RECOGNITION OF IDENTICAL TWINS
}

\author{
Hoda Marouf ${ }^{1}$ and Karim Faez ${ }^{2}$ \\ ${ }^{1}$ Department of Electrical,Computer and Biomedical Engineering, Qazvin branch, \\ IslamicAzad University, Qazvin, Iran \\ ${ }^{2}$ Department of Electrical Engineering, Amirkabir University of Technology, Tehran, \\ Iran
}

\begin{abstract}
Face recognition is one of the most challenging problems in the domain of image processing and machine vision. The face recognition system is critical when individuals have very similar biometric signature such as identical twins. In this paper, new efficient facial-based identical twins recognition is proposed according to geometric moment. The utilized geometric moment is Zernike Moment (ZM) as a feature extractor inside the facial area of identical twins images. Also, the facial area in an image is detected using AdaBoost approach. The proposed method is evaluated on two datasets, Twins Days Festival and Iranian Twin Society which contain scaled and rotated facial images of identical twins in different illuminations. The results prove the ability of proposed method to recognize a pair of identical twins. Also, results show that the proposed method is robust to rotation, scaling and changing illumination.
\end{abstract}

\section{KEYWORDS}

Face Recognition,Identical Twins, Invariant Moment, Zernike Moment

\section{INTRODUCTION}

Human face is considered as a suitable property to identify people from his (her) image. Along with this property, recognition of facial of identical twin is one of the most challenging problems in pattern recognition applications because of the similarity between the pair of twin.

In the domain of facial identical twins recognition, previous works are listed as: in[12], Klare and Jain introduced a face detection algorithm which includes three levels. In the first level, an overall appearance of the face is constructed; in the second level, exact geometric and structural embedment of face with differentiating between two similar faces are performed; and finally, the third level consists of process of skin disorders such as wounds, and so on. Sun et al. [16] utilized CognitecFaceVACS system to recognize identical twins from CASIA Multimodal Biometrics Database and they obtained the true accept rate of approximately $90 \%$ at a false accept rate greater than $10 \%$. Park et al.[14] proposed an identical twins recognition algorithm that consists of three steps: in first step, the proposed method consists of face images which are marked using normal geometric methods; in the second step, the Euclidean distance between a pair of markers are measured and compared; and the final step involves finding the strong similarity on the marked regions. Srinivaset al. [15] studied on distinguishing of twins using marks on the face image. Martin et al. [3] employed DNA approach to recognize identical twins. 
In this paper, we study on a pair of facial images in order to determine whether the images belong to the same person or to a pair of identical twin. For this purpose, we propose the geometric moments to extract feature vector from facial images of twins to recognize identical twins.

This paper is organized as follow: feature extraction step of a face recognition system isintroduced in Section 2. The proposed method is presented in Section 3. Experimentalresults are described in Section 4 and the paper will be concluded in Section 5.

\section{FeATURE ExTraction}

Each face detection system contains four steps: pre-processing, face localization, feature extraction and classification. Feature extraction refers to the extraction of useful information from raw data so that they are suitable for the classification process. The feature extraction stage is characterized by a series of input patterns. The major problem of feature extraction is that it depends on application and feature extraction methods are not public.

Feature extraction methods can be divided into two majors: structural features and statistical features [11][19]. The first group is based on local structure of image. In other words, the structural features deals with local data. Facial change or change in environmental conditions is the major problem for the structural features [7].

In the statistics-based feature extraction techniques, global data is employed to create a set of feature vector elements in order to perform recognition. A mixture of irrelevant data, which are usually part of a facial image, may result in an incorrect set of feature vector elements. Therefore, data that are irrelevant to facial portion such as hair, shoulders, and background should be is regarded in the feature extraction phase [10].The statistics-based feature extraction techniques are Principle Component Analysis (PCA), Legender Moment (LM) [13] and Zernike Moments (ZM) [20]. Legendre functions are Legendre differential equation. The main advantage is that Legendre moments like Legendre basis functions are orthogonal. Legendre moments are independent of each other and are free of data redundancy.In this study, we use ZM to recognize identical twins that are presented in the next Section.

\section{Proposed Method}

The main goal of this paper is to distinguish the identical twins by face recognition. For this purpose, AdaBoost[18] technique is used for face localization step and subimage creation. After that the ZM technique is employed to extract the feature vector from the subimage of test image. After that the feature vectors inside the subimages of all images in dataset are obtained using ZM approach. Finally, comparison between the feature vector of test image and the feature vectors of all images of dataset is done to select the closest image from dataset as the pair of test image. In the next Section, the AdaBoost face detection, ZM and its task of feature vector creation are described.

\subsection{Face Detection Method}

As the mentioned before, face detection step is the second step of this algorithm to recognize identical twins. This step is based on the combing of successively more complex classifiers in a cascade structure using AdaBoost[18]. Furthermore, the AdaBoost technique is used to select a small number of Haar-like features [18]. 
After finding an object in an image as a face candidate, an ellipse is drowning around the main location of face in an image[8]. For this purpose, an ellipse model is constructed using five parameters: $X_{0}$ and $Y_{0}$ are the centers of the ellipse, $\theta$ is the orientation, $\alpha$ and $\beta$ are the minor and the major axes of the ellipse, respectively. Before the calculation of theseparameters, geometric moments are required to describe. The geometric moments of order $p+q$ of a digitalimageare defined as

$$
M_{p q}=\sum_{x} \Sigma_{y} f(x, y) x^{p} y^{q}(1)
$$

where $p, q=0,1,2, \ldots$ and $f(x, y)$ is the grey-scale value of the digital image at $x$ and ylocation. The translation invariant central moments are obtained by placing origin at thecenter of the image:

$$
\mu_{p q}=\Sigma_{x} \Sigma_{y} f(x, y)\left(x-x_{0}\right)^{p}\left(y-y_{0}\right)^{q}(2)
$$

where $x_{0}=\frac{M_{10}}{M_{00}}$ and $x_{0}=\frac{M_{01}}{M_{00}}$ yare the centers of the connected components. Thus, centerof gravity of the connected components is used as the center of the ellipse. The orientationof the ellipse is computed by determining the least moment of inertia [8].

$$
\theta=\frac{1}{2} \arctan \left(\frac{2 \mu_{11}}{\mu_{20}-\mu_{02}}\right)(3)
$$

where $\mu_{p q}$ shows the central moment of the connected components as described in (2). The length of the major and the minor axes of the best-fit ellipse can also be computed byevaluating the moment of inertia. With the least and the greatest moments of inertia of anellipse defined as

$$
\begin{aligned}
& I_{\min }=\Sigma_{x} \Sigma_{y}\left[\left(x-x_{0}\right) \cos \theta-\left(y-y_{0}\right) \sin \theta\right]^{2}(4) \\
& I_{\max }=\Sigma_{x} \Sigma_{y}\left[\left(x-x_{0}\right) \sin \theta-\left(y-y_{0}\right) \cos \theta\right]^{2}(5)
\end{aligned}
$$

Length of the major and the minor axes are calculated from [8] as

$$
\begin{aligned}
& \alpha=\frac{1}{\pi\left[I_{\max }^{3} / I_{\min }\right]^{\frac{1}{8}}}(6) \\
& \beta=\frac{1}{\pi\left[I_{\min }^{3} / I_{\max }\right]^{\frac{1}{8}}}(7)
\end{aligned}
$$

To determine how well the best-fit ellipse approximates the connected components, a distance measure between the connected components and the best-fit ellipse is calculated as follows [8]

$$
\begin{aligned}
\varnothing_{i} & =\frac{P_{\text {inside }}}{\mu_{00}} \\
\varnothing_{o} & =\frac{P_{\text {outside }}}{\mu_{00}}(9)
\end{aligned}
$$

where the $P_{\text {inside }}$ is the number of background points inside the ellipse, $P_{\text {outside }}$ is the number of points of the connected components that are outside the ellipse, and $\mu_{00}$ is the sizeof the 
connected components. After drawing of ellipse, a subimage is made according to theellipse and finally, the $\mathrm{ZM}$ is used to extract features inside the subimage.

\subsection{Zernike Moment (ZM)}

$\mathrm{ZM}$ is geometric-based moment that is a two dimensional function of orthogonal polynomials on the unit disk. The orthogonal moments of $\mathrm{ZM}$ are rotation and scale invariants which are suitable for pattern recognition applications [5][6][8][17]. ZM contains several orthogonal sets of complex-valued polynomials defined as

$$
V_{n m}(x, y)=R_{n m}(x, y) \exp \left(j m \tan ^{-1}\left(\frac{y}{x}\right)\right)
$$

where $x^{2}+y^{2} \leq 1, n \geq 0,|m| \leq n$, and the radial polynomials $\left\{R_{n m}\right\}$ are defined as

$$
R_{n m}(x, y)=\sum_{s=0}^{(n-m) / 2} S_{n,|m| s}\left(x^{2}+y^{2}\right)^{\frac{n-2 s}{2}}
$$

Where

$$
S_{n,|m|, s}=(-1)^{s} \frac{(n-s) !}{s !\left(\frac{n+\mid m}{2}-s\right) !\left(\frac{n-|m|}{2}-s\right) !}
$$

The ZM of order $n$ and repetition $m$ can be computed as

$$
Z M_{n m}=\frac{n+1}{\pi} \sum_{x} \Sigma_{y} f(x, y) V_{n m}^{*}(x, y)(13)
$$

It should be noted that the PZM is computed for positive $m$ because $V_{n m}(x, y)=V_{n m}^{*}(x, y)$.Center of the unit disk is located on the origin of coordinates and so ZM technique is independent of scaling and rotation.In the next Section, ZM approach will be utilized to extract feature vector elements.

\subsection{Creating feature vector}

After face localization and subimage creation, the $\mathrm{ZM}$ is computed for each subimage as face features. The feature vector elements are defined according to ZM orders as

$$
F V_{j}=\left\{Z M_{k m} \mid k=j_{0} j+1, \ldots, N\right\}
$$

where $j$ is interval $[1, N-1]$ and so, $F V_{j}$ contains all the $\mathrm{ZM}$ from order $j$ to $N$. Samples of feature vector elements will be demonstrated in Table 1 for $j=3,5$ and 9 , and $N=10$. As Table 1 shows, increasing of $j$ decreases the number of elements in each feature vector $\left(F V_{j}\right)$.

\section{EXPERIMENTAL RESUltS}

The proposed method is evaluated on two datasets: Twins Days Festival [2] and Iranian Twin Society [1] which contain 520 and 600 pairs of identical twins images, respectively. The used datasets contain the scaled and rotated faces with different illuminations. Figure 1 shows the subimages of some twin test images. The results of identical twins recognition using $\mathrm{ZM}$ is compared with the results of LM [13]. Experiments have been carried out in three steps according 
to order of moment. In the first step, order $n$ is in interval [1,6], in the second step, order $n$ is in interval $[6,8]$ and for third step, order $n$ is in interval $[9,10]$ (Table 2).

In this paper, $N$ is set $10(N=10)$ and $j$ varies from 1 to 9 . The misclassification rate of all geometric moments (LM and $\mathrm{ZM}$ ) is presented in Table 3. The misclassification rate reported in the table are computed as

$$
\text { Error rate }=\frac{\text { No.of misclssification }}{\text { No.of total testing patterns }}
$$

Table 3 shows misclassification rates of LM and ZM. Comparison between geometric moments in Table 3 proves that higher order moments of the $\mathrm{ZM}$ have most information for face recognition while low-order moments have no significant effect on the system error. According to the table, LM achieves high misclassification rate on recognition of twins because the rotation of face in an image has bad effect on the performance of LM. As Table 3 shows, the misclassification rate of $\mathrm{ZM}$ is lower than the $\mathrm{LM}$ because $\mathrm{ZM}$ is rotation and scale invariant.

Table 1.Feature vector elements based on the ZM

\begin{tabular}{|c|c|c|c|}
\hline \multirow{2}{*}{$j$ value } & \multicolumn{2}{|c|}{$\mathrm{FV} \quad$ feature elements $\left(Z M_{k m}\right)$} & \multirow{2}{*}{$\begin{array}{l}\text { Number of } \\
\text { feature element }\end{array}$} \\
\hline & $\mathbf{K}$ & $\mathbf{M}$ & \\
\hline \multirow{7}{*}{4} & 4 & $0,2,4$ & \multirow{7}{*}{30} \\
\hline & 5 & $1,3,5$ & \\
\hline & 6 & $0,2,4,6$ & \\
\hline & 7 & $1,3,5,7$ & \\
\hline & 8 & $0,2,4,6,8$ & \\
\hline & 9 & $1,3,5,7,9$ & \\
\hline & 10 & $0,2,4,6,8,10$ & \\
\hline \multirow{5}{*}{6} & 6 & $0,2,4,6$ & \multirow{5}{*}{24} \\
\hline & 7 & $1,3,5,7$ & \\
\hline & 8 & $0,2,4,6,8$ & \\
\hline & 9 & $1,3,5,7,9$ & \\
\hline & 10 & $0,2,4,6,8,10$ & \\
\hline \multirow{2}{*}{9} & 9 & $1,3,5,7,9$ & \multirow{2}{*}{11} \\
\hline & 10 & $0,2,4,6,8,10$ & \\
\hline
\end{tabular}

Table 2. Feature vector elements produced by geometric moments in each experiment.

\begin{tabular}{|c|l|l||}
\hline Cat. & LM feature elements & ZM feature elements \\
\hline & $n=1, m=1$ & $n=1, m=1$ \\
& $n=2, m=0,2$ & $n=2, m=0,2$ \\
1 & $n=3, m=1,3$ & $n=3, m=1,3$ \\
& $n=4, m=0,2,4$ & $n=4, m=0,2,4$ \\
& $n=5, m=1,3,5$ & $n=5, m=1,3,5$ \\
& $n=6, m=0,2,4,6$ & $n=6, m=0,2,4,6$ \\
\hline \multirow{4}{*}{2} & $n=6, m=0,2,4,6$ & $n=6, m=0,2,4,6$ \\
& $n=7, m=1,3,5,7$ & $n=7, m=1,3,5,7$ \\
& $n=8, m=0,2,4,6,8$ & $n=8, m=0,2,4,6,8$ \\
\hline \multirow{2}{*}{3} & $n=9, m=1,3,5,7,9$ & $n=9, m=1,3,5,7,9$ \\
& $n=10, m=0,2,4,6,8,10$ & $n=10, m=, 0,2,4,6,8,10$ \\
\hline
\end{tabular}


Visual results of ZM on pair of identical twins are illustrated in Figure 2 which refers to Twins Days Festival [2] and Iranian Twin Society [1] datasets, respectively.

According to numerical and visual results, $\mathrm{ZM}$ is able to create informative feature vector inside the subimages of pair of identical twins which is necessary for recognition of identical twins. The results prove that $\mathrm{ZM}$ is scale and rotation invariant.

Table 3. Error rate of each geometric moment in different categories. The bold values means the best values

\begin{tabular}{|c|c|c|c|c|c|c||}
\hline \multirow{2}{*}{ Cat. } & \multicolumn{3}{|c|}{ LM } & \multicolumn{3}{c|}{ ZM } \\
\cline { 2 - 7 } & $\begin{array}{c}\text { No. of } \\
\text { Feature } \\
\text { Elements }\end{array}$ & $\begin{array}{c}\text { No. of } \\
\text { Misclassificati } \\
\text { on }\end{array}$ & Error rate & $\begin{array}{c}\text { No. of } \\
\text { Feature } \\
\text { Elements }\end{array}$ & $\begin{array}{c}\text { No. of } \\
\text { Misclassificati } \\
\text { on }\end{array}$ & $\begin{array}{c}\text { Error } \\
\text { rate }\end{array}$ \\
\hline $\begin{array}{c}\mathrm{n}=1,2, \ldots, \\
6\end{array}$ & 15 & 20 & $10 \%$ & 15 & 17 & $\mathbf{8 . 5 \%}$ \\
\hline $\mathrm{n}=6,7,8$ & 13 & 18 & $9.1 \%$ & 13 & 13 & $\mathbf{6 . 5 \%}$ \\
\hline $\mathrm{n}=9,10$ & 11 & 12 & $6.1 \%$ & 11 & 8 & $\mathbf{4 \%}$ \\
\hline
\end{tabular}
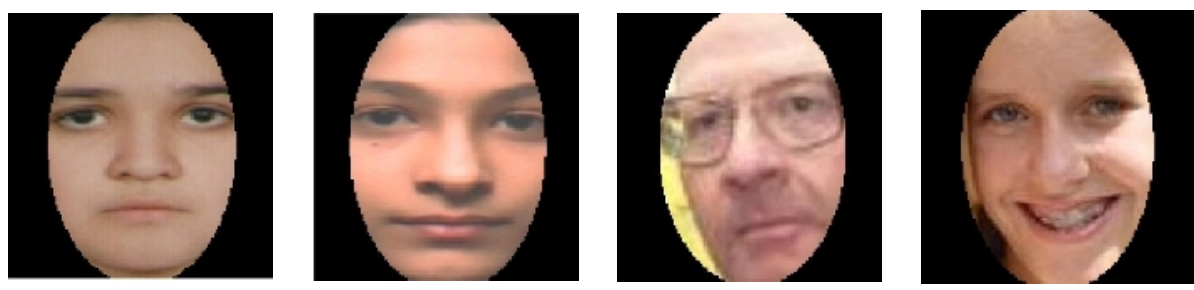

Figure 1. Creating of subimage based on the ellipse formation.

Table 4 shows the second phase of testing where the two geometric moments are compared on finding a pair of a person as the twin ink-nearest persons. In the other words for a test image, his (her) pair is found in $k$-nearest persons. In Table 4 , the above comparison is done in several ranks $(k), k=3,5,7$ and 9. Also, visual results of $\mathrm{ZM}$ on the second phase of testing are demonstrated in Figure 3.The results reported in Table 4 are the percentage of identical twins that the pair ofa person cannot be found in $k$-nearest persons (16). According to the results of ZM in Table 4and Figure 3, pair of input image as the identical twin is detected in 3-nearest persons with the probability of $95.1 \%(100 \%-4.9 \%)$ while with $\mathrm{LM}$, the obtained value is with the probability of $91.3 \%$ (100\%-8.7\%). For the other ranks, the ZM approach takes the best error rates. As a result of Table 4, the detected person as the identical twin using the proposed feature extractor is in $k$ nearest persons with high probability.

$$
\text { Error rate on rank } k=\frac{\text { No.of misclssification samples on rank } k}{\text { No.of total testing patterns }}
$$

\section{CONCLUSIONS}

This paper is focused on the improving of face recognition systems for distinguishing of a pair of identical twins. The proposed method is based on the Zernike Moment (ZM) as a feature extractor to recognize a pair of (identical or non-identical) twins. Also, the location of the face in an image is detected using the AdaBoost method and then the ZM method is utilized to construct feature vector elements. Experimental results on two datasets show that the proposed method is superior 
to the other geometric moment such as Legendre Moment (LM) and also is robust to rotation and scaling and changing illumination.
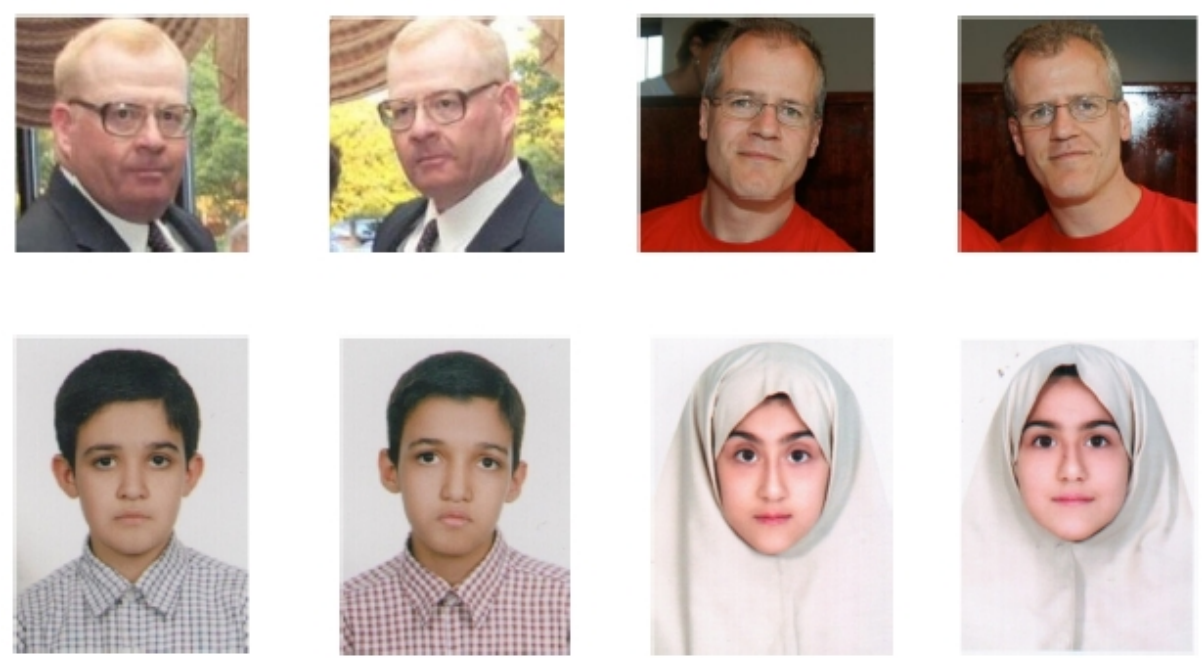

Figure 2. Samples of testing identical twins which were correctly classified by ZM. The first row refers to the results of ZM on the Twins Days Festival dataset [2] and the second row is the results of ZM on the Iranian Twin Society dataset [1].

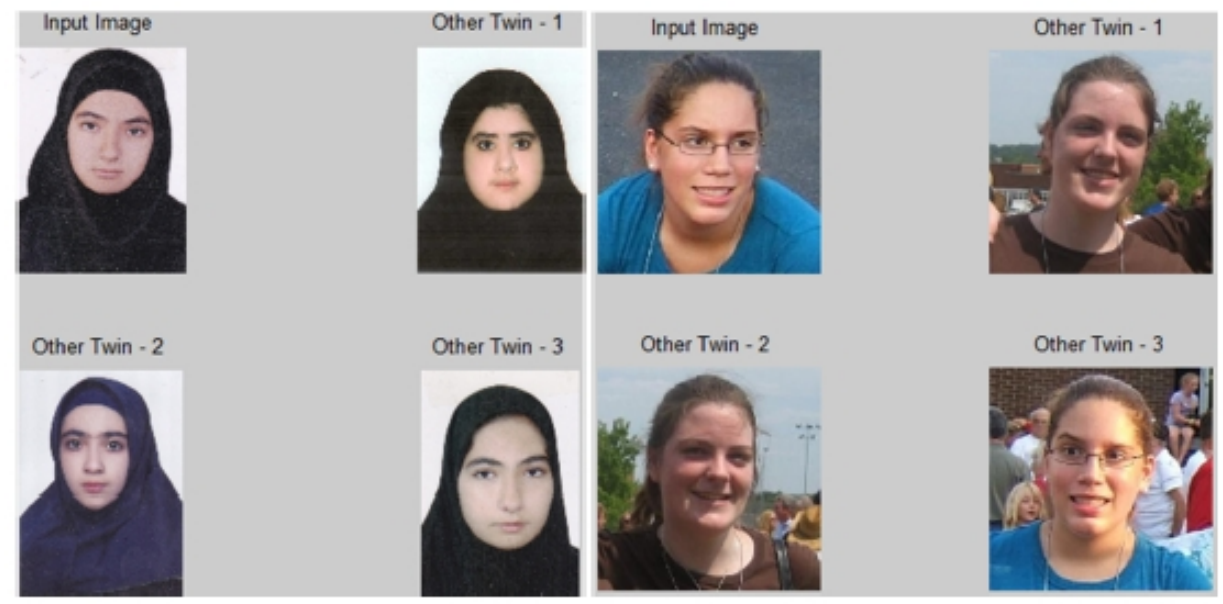

Figure 3. Visual results of $\mathrm{ZM}$ on the second phase of testing with rank=3.

Table 4. Results of geometric moments on the second phase of testing with rank=3. Bold values refer to the best scores.

\begin{tabular}{|c|c|c|}
\hline \multirow{2}{*}{ Rank } & \multicolumn{2}{|c|}{ Feature extractor } \\
\cline { 2 - 3 } & LM & $\mathbf{Z M}$ \\
\hline 3 & $8.7 \%$ & $\mathbf{4 . 9} \%$ \\
\hline 5 & $5.3 \%$ & $\mathbf{1 . 2} \%$ \\
\hline 7 & $3.2 \%$ & $\mathbf{0} \%$ \\
\hline 9 & $0.9 \%$ & $\mathbf{0} \%$ \\
\hline
\end{tabular}




\section{REFERENCES}

[1] Iranian twin society. http://www.irtwins.com/.

[2] Twins days festival. http://www.twinsdays.org.

[3] Arias, E., MacDorman, M.F., Strobino, D.M.\&Guyer, B. (2003)“Annual summary of vitalstatistics2002”,Pediatrics,Vol. 112, No. 6, pp. 1215-1230.

[4] Bailey, R.R. (1993)“Automatic recognition of handwritten numerals via orthogonal momentsusing statistical and neural network classifiers”, Ph.D. thesis, Dallas, TX, USA (1993),aAI9331109.

[5] Belhumeur, P., Hespanha, J.\&Kriegman, D. (1997)"Eigenfaces vs. fisherfaces recognition using classspecific linear projection", IEEETransactions on Pattern Analysis and Machine Intelligence,Vol. 19, No. 7, pp. 711-720.

[6] Belkasim, S., Shridhar, M.\&Ahmadi, M. (1991)“Pattern recognition with moment invariants:A comparative study and new results”,Pattern Recognition,Vol. 24, No.12, pp. 1117 1138,http://www.sciencedirect.com/science/article/pii/003132039190140Z.

[7] Bichsel, M.\&Pentland, A. (1994)“Human face recognition and the face image sets topology" CVGIP Image Understanding,Vol. 59, No. 2, pp. 254-261.

[8] Haddadnia, J., Ahmadi, M.\&Faez, K. (2002)“An efficient method for recognition of human facesusing higher orders pseudo Zernike moment invariant”,In:Proceedings. Fifth IEEE International Conference on Automatic Face and GestureRecognition 2002, pp. 330-335.

[9] Haddadnia, J., Faez, K.\&Moallem, P. (2001)“Neural network based face recognition with moment invariants",InProceedings of Int. Conference on Image Processing 2001, Vol. 1, pp. 1018-1021.

[10] Haddadnia, J., Ahmadi, M.\&Faez, K. (2003)“An efficient feature extraction method withpseudoZernike moment in RBF neural network-based human face recognition system”, EURASIP Journal of Appl. Signal Process,pp. 890-901, http://dx.doi.org/10. 1155/S1110865703305128.

[11] Hjelms, E.\& Low, B.K. (2001)“Face detection: A survey",Computer Vision and Image Understanding, Vol. 83, No. 3, PP. 236-274.

[12] Klare, B.\& Jain, A. (2010)“On a taxonomy of facial features”,InFourth IEEE International Conference on Biometrics, Theory Applicationsand Systems (BTAS), pp. 1-8.

[13] Liao, S.\&Pawlak, M. (1996)“'On image analysis by moments”,IEEE Transactions onPattern Analysis and MachineIntelligence, Vol. 18, No. 3, pp. 254-266.

[14] Park, U., Jillela, R., Ross, A.\& Jain, A. (2011)“Periocular biometrics in the visible spectrum”, IEEE Transactions onInformation Forensics and Security, Vol. 6 No. 1, pp. 96-106.

[15] Srinivas, N., Aggarwal, G., Flynn, P.\&VorderBruegge, R. (2012)“Analysis of facial marks todistinguish between identical twins",IEEE Transactions onInformation Forensics and Security, Vol. 7, No. 5, pp. 1536-1550.

[16] Sun, Z., Paulino, A.A., Feng, J., Chai, Z., Tan, T.\& Jain, A.K.(2010) “A study of multibiometrictraits of identical twins",http://dx.doi.org/10.1117/12.851369.

[17] Teh, C.H.\& Chin, R.(1988) "On image analysis by the methods of moments". IEEE Transactions onPattern Analysisand Machine Intelligence, Vol. 10, No. 4, pp. 496-513.

[18] Viola, P.\& Jones, M. (2001)"Rapid object detection using a boosted cascade of simple features", InProceedings of the IEEE Computer Society Conference on Computer Vision and Pattern Recognition 2001 (CVPR'01),Vol. 1, pp. I-511-I-518.

[19] Yang, M.H., Kriegman, D., Ahuja, N.(2002) “Detecting faces in images: a survey”,IEEE Transactions onPatternAnalysis and Machine Intelligence, Vol. 24, No. 1, pp. 34-58.

[20] Zernike, v.F.(1934), "Beugungstheorie des schneidenver-fahrens und seiner verbesserten form,der phasenkontrastmethode”,Physica,Vol. 1, No. 7, pp. 689-704. 